\title{
Development, validation, and comparison of four methods to simultaneously quantify L-arginine, citrulline, and ornithine in human plasma using hydrophilic interaction liquid chromatography and electrospray tandem mass spectrometry
}

\author{
Xianyin Laia,b*, Jeffrey A. Kline ${ }^{b, c}$, Mu Wang ${ }^{a}$ \\ a Department of Biochemistry and Molecular Biology, Indiana University School of \\ Medicine, Indianapolis, IN 46202, USA \\ ${ }^{b}$ Department of Cellular \& Integrative Physiology, Indiana University School of \\ Medicine, Indianapolis, IN 46202, USA \\ c Department of Emergency Medicine, Indiana University School of Medicine, \\ Indianapolis, IN 46202, USA
}

Correspondence: Xianyin Lai, Ph.D.

Department of Biochemistry and Molecular Biology

Department of Cellular and Integrative Physiology

Indiana University School of Medicine

635 Barnhill Drive, Room 0044

Indianapolis, IN 46202 USA

Email: xlai@iu.edu

Phone: 317-278-0084

Fax: $\quad 317-274-4686$

Keywords: Arginine, Citrulline, Ornithine, HILIC-MS/MS, Plasma, Validation

This is the author's manuscript of the article published in final edited form as:

Lai, X., Kline, J. A., \& Wang, M. (2015). Development, validation, and comparison of four methods to simultaneously quantify l-arginine, citrulline, and ornithine in human plasma using hydrophilic interaction liquid chromatography and electrospray tandem mass spectrometry. Journal of Chromatography B, 1005, 47-55. http://doi.org/10.1016/j.jchromb.2015.10.001 



\section{ABSTRACT:}

To understand the role of L-arginine depletion in impaired nitric oxide synthesis in disease, it is important to simultaneously quantify arginine, citrulline, and ornithine in the plasma. Because the three amino acids are endogenous analytes, true blank matrix for them is not available. It is necessary and valuable to compare the performance of different approaches due to lack of regulatory clarity for validation. A two-step sample preparation method using methanol as protein precipitation reagent was developed in this study is used for sample preparation. Because true blank matrix for endogenous analytes is not available, water as blank matrix, 1\% BSA in PBS as blank matrix, surrogate analyte, and background subtraction were designed to establish successful quantification methods. Four methods to simultaneously quantify arginine, citrulline, and ornithine in human plasma using hydrophilic interaction liquid chromatography and electrospray tandem mass spectrometry were developed, validated, and compared. The developed two-step sample preparation method using methanol as protein precipitation reagent in this study needs less time and provides higher recovery comparing with other approaches. Three of the four methods, water as blank matrix, 1\% BSA in PBS as blank matrix, and surrogate analyte, have been successful in fulfilling all the criteria, while background subtraction has failed. Results of the measured concentrations in 97 human plasma samples using the three methods show that the difference between any two methods or among the three methods presents $100 \%$ of samples with less than $20 \%$ for all the three amino acids and majority of them are under 10\%. The developed two-step sample preparation method using methanol as protein precipitation reagent is simple and convenient. Three of the four methods are fully validated and the validation is 
successful. The BSA functioned effectively as a blank matrix for these three amino acids, considering cost, data quality, matrix similarity, and practicality. 


\section{Introduction}

This work measures L-arginine in plasma from patients with acute pulmonary embolism (PE). L-arginine is the primary substrate used by endothelial nitric oxide synthase to produce nitric oxide (NO) and L-citrulline. Nitric oxide then diffuses to the vascular smooth muscle, where it functions as the primary vasodilator of pulmonary vasculature; disease conditions that impair the normal steady-state delivery of NO secondarily allow for contraction of vascular smooth muscle. L-arginine can be depleted by either decreased intake or increased destruction, primarily by the action of circulating arginase-I (abundant in erythrocytes), which cleaves L-arginine to form ornithine and urea. Thus, the simultaneous measurement of L-arginine, citrulline and ornithine and their respective ratios, provides mechanistic insight into the cause of L-arginine depletion and subsequent NO lack. Prior work in animals and humans has found that acute pulmonary embolism (PE) causes hemolysis, leading to increased plasma arginase concentrations, with reduced L-arginine concentrations, leading to impaired NO synthesis and pulmonary vasopasm [1-4]. In the United States, approximately 200,000 individuals are diagnosed with acute pulmonary embolism (PE) each year, resulting in substantial morbidity, primarily related to damage to the right ventricle [5]. Patients with severe tricuspid regurgitation, implying more severe PE and more hemolysis appear to be at the highest risk of acute L-arginine depletion, and persistent right ventricular dysfunction $[6,7]$.

Numerous quantitative methods have been developed and validated to determine a single amino acid of the three [8]. However, fewer methods have been 
developed and validated for simultaneously quantification of any two of the three amino acids $[9,10]$. Among various methods, hydrophilic interaction liquid chromatography (HILIC) columns are gaining popularity due to their simplicity, not requiring traditional derivatization steps or ion-pairing separations for amino acid separation. Currently, only one article has been reported to simultaneously quantify all the three amino acids in plasma or serum using a HILIC column [11]. However, the published method has not been fully validated.

Because the three amino acids are endogenous analytes, true blank matrix for them is not available. Approaches using a surrogate matrix, background subtraction, or a surrogate analyte have been utilized to establish quantification methods for endogenous analytes in different projects [12-14]. It is necessary and valuable to compare these approaches due to lack of regulatory clarity for validation.

In this study, several approaches have been utilized to establish successful quantification methods. Surrogate matrix is the first choice applied in many projects [15]. Water and BSA are the most commonly used surrogate matrix. When the plasma with endogenous analytes is used as blank matrix, two strategies have been applied: surrogate analyte approach by spiking of a stable isotope-labeled the analyte as a surrogate standard and background subtraction technique during data processing to handle the spiking of exogenous levels of the analyte. Due to the debate on which approach should be utilized and lack of regulatory clarity for validation of endogenous analytes, we compared four methods: water as blank matrix, 1\% BSA in PBS as blank matrix, surrogate analyte, and background subtraction. 
In summary, we report the development, validation, and comparison of four methods to simultaneously quantify arginine, citrulline, and ornithine in human clinical plasma using HILIC and tandem mass spectrometry (MS/MS). The established method is expected to be rapid and robust, enabling efficient analysis of large number of clinical plasma samples.

\section{Materials and methods}

\subsection{Reagents}

L-arginine (99.0\%, AR01), L-citrulline (98.0\%, Cl01), L-ornithine: $\mathrm{HCl}(99.0 \%$, OR01), and BSA were purchased from Sigma-Aldrich (St. Louis, MO, USA). Larginine: $\mathrm{HCl}$ (guanido-15N2, 98.7\%, AR21), L-arginine: $\mathrm{HCl}$ (13C6, 98.0\%, AR61), Lcitrulline (5,5-D2, 99.3\%, Cl21) , L-citrulline (4,4,5,5-D4, 98.0\%, Cl41), L-ornithine: $\mathrm{HCl}$ (5,5-D2, 98.0\%, OR21), and L-ornithine: $\mathrm{HCl}(3,3,4,4,5,5-\mathrm{D} 6,98.9 \%$, OR61) were obtained from Cambridge Isotope Laboratories (Tewksbury, MA). AR61, Cl21, and OR21 were used as internal standards. AR21, Cl41, and OR61 were used as surrogate analytes in the surrogate analyte approach. Ammonium formate was purchased from Santa Cruz Biotechnology (Dallas, TX). Phosphate Buffered Saline (PBS-1X) was obtained from Lonza (Allendale, NJ). LC-MS grade water $\left(\mathrm{H}_{2} \mathrm{O}\right)$, LC-MS grade $0.1 \%$ formic acid in acetonitrile (ACN), LC-MS grade ACN, and $0.1 \%$ formic acid in water $\left(\mathrm{H}_{2} \mathrm{O}\right)$ were purchased from Burdick \& Jackson (Muskegon, MI, USA). Isopropyl alcohol and methanol (HPLC grade) were purchased from Fisher Scientific (Waltham, MA). The 
details of the name, abbreviation, labelling, and chemical structure were listed in Supplemental Method 1.

\subsection{Patient samples}

This study was approved by the Indiana University Institutional Review Board (IRB \# 1208009308). Patients were enrolled in an ongoing randomized, double-blind, placebo-controlled clinical trial (NCT01939301) to test the therapeutic efficacy of inhaled nitric oxide. Patients all had confirmed acute pulmonary embolism, diagnosed on the basis of contrast-enhanced computed tomography of the chest. All patients had evidence of right ventricular strain, usually with severe TR, and were treated with systemic heparin anticoagulation. Plasma was drawn by a qualified phlebotomist with care to avoid hemolysis. Blood for the current assay was additionally anticoagulated by addition of EDTA to a final concentration of $50 \mathrm{mmol} / \mathrm{L}$. Within $30 \mathrm{~min}$ of blood draw, plasma samples were centrifuged at $4{ }^{\circ} \mathrm{C}$ and at $10,000 \mathrm{rcf}$ for $20 \mathrm{~min}$ and aliquots of supernatants were stored at $-80{ }^{\circ} \mathrm{C}$ until sample preparation for LC-MS/MS analysis.

\subsection{Sample preparation}

The details of the preparation of standards and quality control samples were described in Supplemental Method 2. Samples were prepared as follows: A $50 \mu \mathrm{L}$ of each sample was spiked with $10 \mu \mathrm{L}$ of $200 \mu \mathrm{M}$ internal standard and incubated at room temperature for $10 \mathrm{~min}$. The samples were extracted with $540 \mu \mathrm{L}$ of methanol and 
agitated in an Eppendorf Thermomixer at 1,400 rpm for $3 \mathrm{~min}$ followed by centrifugation at 14,000 rcf. $120 \mu \mathrm{L}$ of supernatant was transferred to a vial and $80 \mu \mathrm{L}$ of $5 \mathrm{mM}$ ammonium formate were added into the vial. The samples were ready for analysis.

\subsection{Instrumentation}

A Dionex Ultimate 3000 UHPLC system (Sunnyvale, CA) consists of a pump, autosampler, column oven, and UV detector. A SCIEX 4000 QTRAP triple quadrupole mass spectrometer (Toronto, Canada) with a TurbolonSpray probe was used in positive ion mode. SCIEX Analyst 1.5 was used for data collection and SCIEX MutiQuant 3.0.1 was used for peak integration and concentration calculation.

\subsection{Chromatographic conditions}

HPLC separation was performed on a Phenomenex Kinetex HILIC 100A column $(4.6 \times 100 \mathrm{~mm}, 2.6 \mu \mathrm{m})($ Torrance, $\mathrm{CA})$ at $30^{\circ} \mathrm{C}$. Mobile phase A was $5 \mathrm{mM}$ ammonium formate with $0.1 \%$ formic acid in water and B was ACN with $0.1 \%$ formic acid. The gradient program was $0.0-0.5 \mathrm{~min}, 70 \% \mathrm{~B} ; 0.5-1.0 \mathrm{~min}$, gradient to $40 \% \mathrm{~B} ; 1.0$ $3.5 \mathrm{~min}, 40 \% \mathrm{~B}$; 3.5-4.0 min, gradient to $70 \% \mathrm{~B}$; and 4.0-6.0 min, $70 \% \mathrm{~B}$. The flow rate was $0.6 \mathrm{~mL} / \mathrm{min}$ during all separation steps and injection volume was $10 \mu \mathrm{L}$.

\subsection{Validation procedure}


The validations were performed according to the Guidance for Industry: Bioanalytical Methods Validation issued by the U.S. Department of Health and Human Services, the Food and Drug Administration, the Center for Drug Evaluation and Research (CDER), and the Center for Veterinary Medicine (CVM) in May 2001. The details were described in Supplemental Method 3.

\section{Results and discussions}

\subsection{Sample preparation}

Measuring plasma amino acids including arginine, citrulline, and ornithine have been reported with various sample preparation methods based on types of sample and analytical column, especially the analytical column selection. Currently, HILIC columns are gaining popularity due to their simplicity, not requiring traditional derivatization steps or ion-pairing separations for amino acid separation. To analyze one, two, or all of the three amino acids in plasma or serum in one injection using a HILIC column, several sample preparation approaches have been previously reported $[10,11,16]$. However,

two issues were observed when the reported methods were followed during our method development. One of them was the choice of the reagent for protein precipitation. The other was the simplicity of sample preparation approaches. Acetonitrile, methanol, and isopropanol are the most commonly used protein precipitation reagents. When acetonitrile was applied initially in the development, it was found that the recovery of all three amino acids was low, especially for ornithine. Therefore, an investigation of 
reagent type and volume was carried out to obtain better recovery for all the three amino acids. Arginine (AR21), citrulline (Cl41), ornithine (OR61), and their internal standards (AR61, Cl21, and OR21) were spiked into $50 \mu \mathrm{L}$ of human plasma. Multiple volumes (two, three, four, five, seven, nine, and eleven times of $50 \mu \mathrm{L}$ ) of acetonitrile, methanol, and isopropanol were used for protein precipitation and the amino acid extraction. One fifth of the supernatant was transferred to a vial. Eighty $\mu \mathrm{L}$ of $5 \mathrm{mM}$ ammonium formate were added into the vial, followed by adding certain amount of acetonitrile, methanol, or isopropanol to reach the final volume of $200 \mu \mathrm{L}$. Ten $\mu \mathrm{L}$ of each sample were injected for comparison. The results in Figure 1 indicates that reagent type is a greater factor than volume for the recovery. Around five times of the plasma volume, the performance for arginine is methanol > isopropanol > acetonitrile, for citrulline is methanol $\geq$ isopropanol $>$ acetonitrile, and for ornithine is methanol > isopropanol $\geq$ acetonitrile. However, volume is a critical factor as well. The performance of acetonitrile dramatically decreases with the increase of its volume for all the three amino acids. The performance of isopropanol slightly decreases with the increase of its volume all the three amino acids. On the contrary, the performance of methanol stays stable with the increase of its volume all the three amino acids. Based on the performance and consideration of next steps in the sample preparation, $540 \mu \mathrm{L}$ of methanol (about eleven times of the plasma volume) was chosen for protein precipitation and amino acid extraction. The extraction step was followed by a dilution step. Basically, the developed method is a two-step sample preparation approach. In numerous publications, dryness and resuspension steps are often involved, which 
requires additional process. In comparison, the two-step sample preparation is simple and superior to other approaches.

\subsection{MS/MS detection}

Choosing product ions of the three amino acids has been diverse in literature. The product ions of arginine have been chosen as 43.0, 60.0, and $70.0[11,12,17,18]$, the product ions of citrulline have been chosen as $70.1,113.0$, and $159.1[11,12,17$, 18], and the product ions of ornithine have been chosen as 70.0 and $116.1[11,17,18]$. Theoretically, multiple product ions are available for transition monitoring. As long as a transition passes the criteria in a full validation, especially the selectivity and LLOQ, the transition is acceptable. Transitions, retention times, and ionization source parameters for each analyte were listed in Supplemental Method 1. Representative chromatograms of the current LC-MS/MS analysis using the chosen transitions from a patient sample are shown in Figure 2.

\subsection{Calibration curve and linearity}

The assay validation has been designed to carry out using four methods: water as blank matrix, 1\% BSA in PBS as blank matrix, surrogate analyte, and background subtraction. The calibration curves from three consecutive batches using water as blank matrix showed an overall accuracy of $98.3-100.8 \%$ with RSD of less than $3.7 \%$ for arginine, $99.2-101.0 \%$ with RSD of less than $2.9 \%$ for citrulline, and $97.3-102.2 \%$ 
with RSD of less than $2.5 \%$ for ornithine. The detailed results are shown in Supplemental Table 1. The calibration curves from three consecutive batches using BSA as blank matrix showed an overall accuracy of $95.3-104.8 \%$ with RSD of less than 2.6\% for arginine, $98.7-102.6 \%$ with RSD of less than $4.1 \%$ for citrulline, and $97.3-$ $103.1 \%$ with RSD of less than $3.8 \%$ for ornithine. The detailed results are shown in Table 1 and Supplemental Table 2. The calibration curves from three consecutive batches using surrogate analytes showed an overall accuracy of $96.8-104.1 \%$ with RSD of less than $3.9 \%$ for arginine, $94.5-107.5 \%$ with RSD of less than $6.2 \%$ for citrulline, and $98.6-103.3 \%$ with RSD of less than $3.1 \%$ for ornithine. The detailed results are shown in Supplemental Table 3. The linear ranges of the three amino acids using the first three methods are $4-200 \mu \mathrm{M}$, covering the reported clinical concentration levels [11].

The last method, background subtraction, encountered a great challenge. The endogenous levels of arginine, citrulline, and ornithine in the human plasma serving as blank matrix are $154.4,17.0$, and $63.1 \mu \mathrm{M}$, respectively. Fifteen percent of the endogenous levels are 23.2, 2.6, and $9.5 \mu \mathrm{M}$. Spiking any amount of standards below the $15 \%$ of the endogenous level may not even produce any significant peak area changes, since the requirement of precision is RSD $\leq 20.0 \%$ at LLOQ and $\leq 15.0 \%$ at any other concentration level. Results show that the linear ranges of arginine, citrulline, and ornithine using the background subtraction method are $25-200,4-200$, and 10-200 $\mu \mathrm{M}$, respectively. Comparing to the other three methods, the background subtraction method exhibits a limited linear range. The calibration curves from three consecutive batches using the background subtraction showed an overall accuracy of $98.3-103.6 \%$ with 
RSD of less than $6.1 \%$ for arginine, $95.0-105.0 \%$ with RSD of less than $4.5 \%$ for citrulline, and $97.2-101.8 \%$ with RSD of less than $6.9 \%$ for ornithine. The detailed results are shown in Supplemental Table 4.

\subsection{Precision and accuracy}

The intra- and inter-assay precision and accuracy from the three consecutive batches using water as blank matrix are shown in Supplemental Table 5. The intraassay accuracy is $88.8-99.3 \%$ with RSD of $1.1-3.3 \%$ for arginine, $92.5-101.5 \%$ with RSD of $0.6-2.9 \%$ for citrulline, and $91.9-102.1 \%$ with RSD of $1.2-3.5 \%$ for ornithine. The inter-assay accuracy is $90.3-95.3 \%$ with RSD of $1.8-4.3 \%$ for arginine, $95.3-$ $99.8 \%$ with RSD of $1.6-3.8 \%$ for citrulline, and $94.5-98.6 \%$ with RSD of $2.0-3.8 \%$ for ornithine. The intra- and inter-assay precision and accuracy from the three consecutive batches using BSA as blank matrix are shown in Table 2 and Supplemental Table 6. The intra-assay accuracy is $90.5-108.0 \%$ with RSD of $1.3-3.2 \%$ for arginine, $95.7-$ $105.9 \%$ with RSD of $1.1-4.4 \%$ for citrulline, and $94.7-104.1 \%$ with RSD of $1.1-2.7 \%$ for ornithine. The inter-assay accuracy is $90.3-95.3 \%$ with RSD of $1.8-4.3 \%$ for arginine, 96.6-103.8\% with RSD of $1.4-5.2 \%$ for citrulline, and $95.1-102.8 \%$ with RSD of $1.8-$ 3.0\% for ornithine. The intra- and inter-assay precision and accuracy from the three consecutive batches using surrogate analytes are shown in Supplemental Table 7 . The intra-assay accuracy is $87.2-101.8 \%$ with RSD of $1.1-3.4 \%$ for arginine, $89.9-106.9 \%$ with RSD of $1.0-5.0 \%$ for citrulline, and $90.9-105.0 \%$ with RSD of $0.8-3.4 \%$ for ornithine. The inter-assay accuracy is $88.2-99.9 \%$ with RSD of $2.2-2.9 \%$ for arginine, 
90.3-103.8\% with RSD of $2.0-4.2 \%$ for citrulline, and $91.4-102.4 \%$ with RSD of $1.6-$ $3.0 \%$ for ornithine.

When the background subtraction method was applied, all QC levels including LLOQ of citrulline fulfilled the requirement, but only high and mid QCs of arginine and ornithine met the criteria. The detailed results are shown in Supplemental Table 8. The precision and accuracy from three consecutive batches show an intra-assay accuracy of 91.1-101.7\% with RSD of $4.0-10.4 \%$ for arginine, $91.3-105.8 \%$ with RSD of less than $1.6-14.0 \%$ for citrulline, and $88.8-99.4 \%$ with RSD of $2.3-6.0 \%$ for ornithine. The interassay accuracy is $92.4-101.3 \%$ with RSD of $4.7-8.4 \%$ for arginine, $97.4-100.3 \%$ with RSD of $2.2-12.4 \%$ for citrulline, and $94.8-96.1 \%$ with RSD of $4.2-6.3 \%$ for ornithine.

The precision and accuracy of dilution QCs are shown in Supplemental Tables 9-12. An accuracy of $93.5 \%$ with RSD of $1.7 \%$ for arginine, $98.9 \%$ with RSD of $0.5 \%$ for citrulline, and $101.4 \%$ with RSD of $0.7 \%$ for ornithine were achieved when water was used as blank matrix. An accuracy of $91.9 \%$ with RSD of $0.9 \%$ for arginine, $96.7 \%$ with RSD of $1.5 \%$ for citrulline, and $98.6 \%$ with RSD of $1.3 \%$ for ornithine were achieved when BSA was used as blank matrix. An accuracy of $86.5 \%$ with RSD of $1.1 \%$ for arginine, $86.4 \%$ with RSD of $1.2 \%$ for citrulline, and $86.4 \%$ with RSD of $1.2 \%$ for ornithine were achieved when the surrogate analytes were applied. An accuracy of 90.3\% with RSD of $5.4 \%$ for arginine, $98.9 \%$ with RSD of $0.7 \%$ for citrulline, and $99.8 \%$ with RSD of 3.3\% for ornithine were achieved when the background subtraction was utilized.

\subsection{Selectivity}


Using the first three methods, all the tested six lots of blank matrix samples showed that interference peaks at the retention time of interest either did not exist or satisfied the criteria. Selectivity LLOQs using water as blank matrix obtained an accuracy of $96.0 \%$ with an RSD of $2.5 \%$ for arginine, $102.0 \%$ with an RSD of $1.8 \%$ for citrulline, and $106.2 \%$ with an RSD of $4.9 \%$ for ornithine. Selectivity LLOQs using BSA as blank matrix obtained an accuracy of $105.6 \%$ with an RSD of $5.5 \%$ for arginine, $108.7 \%$ with an RSD of $6.3 \%$ for citrulline, and $106.2 \%$ with an RSD of $4.9 \%$ for ornithine. Selectivity LLOQs using surrogate analytes obtained an accuracy of $90.0 \%$ with an RSD of 3.6\% for arginine, $96.2 \%$ with an RSD of $4.1 \%$ for citrulline, and $101.9 \%$ with an RSD of $2.4 \%$ for ornithine. The detailed results are shown in Supplemental Table 13-15. When the background subtraction method was applied, the selectivity assessment was not applied, because the blank samples had high endogenous levels.

\subsection{Matrix effect}

The detailed matrix effect results are shown in Supplemental Table 16-18. Matrix effect LOQs using water as blank matrix exhibit $0.9 \%$ for arginine, $0.5 \%$ for the internal standard of arginine, 1.4\% for citrulline, 1.4\% for the internal standard of citrulline, $2.0 \%$ for ornithine, and $2.0 \%$ for the internal standard of ornithine. Matrix effect LOQs using BSA as blank matrix exhibit $7.7 \%$ for arginine, $6.2 \%$ for the internal standard of arginine, $-53.9 \%$ for citrulline, $-53.8 \%$ for the internal standard of citrulline, $7.1 \%$ for ornithine, and $2.7 \%$ for the internal standard of ornithine. Matrix effect LOQs using surrogate 
analytes exhibit $-24.3 \%$ for arginine, $-25.3 \%$ for the internal standard of arginine, $58.3 \%$ for citrulline, $-58.4 \%$ for the internal standard of citrulline, $-9.9 \%$ for ornithine, and $-10.1 \%$ for the internal standard of ornithine. All six lots of matrix have showed similar matrix effect for both analytes and their corresponding internal standards. When the background subtraction method was applied, the matrix effect evaluation was not applied, because the blank samples had high endogenous levels.

\subsection{Recovery}

Overall recoveries from low, mid, and high QCs using water as blank matrix were 93.1\% for arginine, $95.4 \%$ for the internal standard of arginine, $93.8 \%$ for citrulline, 91.2\% for the internal standard of citrulline, $93.1 \%$ for ornithine, and $91.4 \%$ for the internal standard of ornithine. The detailed results are shown in Supplemental Table 19. Overall recoveries from low, mid, and high QCs using BSA as blank matrix were $85.0 \%$ for arginine, $91.5 \%$ for the internal standard of arginine, $88.0 \%$ for citrulline, $93.8 \%$ for the internal standard of citrulline, $83.3 \%$ for ornithine, and $90.4 \%$ for the internal standard of ornithine. The detailed results are shown in Table 3. Overall recoveries from low, mid, and high QCs using surrogate analytes were $74.7 \%$ for arginine, $78.6 \%$ for the internal standard of arginine, $85.8 \%$ for citrulline, $91.5 \%$ for the internal standard of citrulline, $78.5 \%$ for ornithine, and $83.5 \%$ for the internal standard of ornithine. The detailed results are shown in Supplemental Table 20. The results indicate that consistent recoveries at all three QC levels of all analytes and their internal standard have been achieved. When the background subtraction method was applied, the 
selectivity assessment was not applied, because of the high endogenous levels in blank samples.

\subsection{Stability}

The stability results of the analytes in BSA under different conditions are shown in Table 4. The accuracy and precision of three freeze/thaw cycles, $48 \mathrm{~h}$ room temperature storage, and 55 days storage at -60 to $-80 \circ \mathrm{C}$ fit the $\pm 15 \%$ criteria. Additionally, stability of $72 \mathrm{~h}$ processed-sample reinjection, 56 days intermediate standard solutions, and 57 days stock standard solutions stored at $2-8 \cdot \mathrm{C}$ were examined. All results demonstrate that the analytes are stable. The stability results of the analytes in water and human plasma under different conditions are shown in Supplemental Tables 21 and 22.

\subsection{Comparison of the four methods}

The four methods, water as blank matrix, 1\% BSA in PBS as blank matrix, surrogate analyte, and background subtraction, have been fully validated. The first three methods have been successful in fulfilling all the criteria, while the last one has failed in LLOQs, LOQs, and low concentrations of the calibration curves. The reason is that the blank samples have high endogenous levels. This is one of the two significant issues in the application of background subtraction. It is extremely difficult to obtain a plasma sample that contains the lowest levels of the endogenous analyte. Assuming such a 
sample as blank matrix is available, any other samples whose endogenous level near the blank matrix sample still face the challenge of accurate quantification, because its LLOQ may not cover these samples. The other significant issue in the application of background subtraction is data processing. Currently, MultiQuant is able to calculate the exogenous level of a spiked analyte by subtracting the endogenous level of the analyte of choice, but considering the endogenous level of the analyte as zero. It works for validation, since exogenous spiking is applied. However, it is not able to obtain the real endogenous level of analytes, because the endogenous level in every sample is subtracted by the endogenous level in the matrix severing as a blank sample, showing that any assay sample having an endogenous level equal or less than the endogenous level in the matrix will be assigned zero or a negative value after the calculation. Some software may be able to process the data differently without zeroing the matrix level. However, these software are often limited with certain mass spectrometers. Crossing different platforms or data formats commonly is not available for regular users. Therefore, background subtraction is the last choice of methods for analyzing endogenous analytes.

On the contrary, the first three methods are easily practicable without considering the two issues described above. However, they are still different from each other with advantages and disadvantages. To compare them, cost, data quality, and matrix similarity are considered. Because surrogate analyte method requires additional stable isotope-labeled standards, it is more expensive than the other two methods. When the surrogate analyte method is applied, three transitions need to be monitored rather than two transitions to monitor in the other two methods. Therefore, less scan time is spent 
on each transition, leading to less accurate peak intensity, especially for low abundance analytes. When multiple analytes are under detection at the same retention time, an additional transition in the three transitions may become a burden. For matrix similarity, the surrogate analyte method has the identical matrix as endogenous analyte does, water is the most unlike matrix, and BSA is in the middle. Generally speaking, BSA is the number one choice, water is the second choice, and the surrogate analyte method is the last one based on the three factors. If cost is not a burden and a high performance mass spectrometer is available with faster scan speed, the surrogate analyte method seems to be a good choice. However, researchers have to examine whether same amount of analyte and surrogate analyte have the same signal response on a mass spectrometer. Unlike exogenous analysis that uses an identical matrix and analyte, endogenous analysis has to use either a surrogate matrix or a surrogate analyte. Therefore, there is no universal rule on whether surrogate matrix or surrogate analyte should be applied. Researchers should consider the cost of labelled analytes, the performance of a mass spectrometer, and the type of matrix comprehensively. A comparison of surrogate matrix and surrogate analyte methods for quantitation of endogenous biomolecules concludes that both assays are well within tolerances prescribed by regulatory guidance for validation, the surrogate analyte approach allows for facile method development, and the surrogate matrix method has the long-term advantage of simplified sample analysis [15]. Another comparison shows that the surrogate analyte in authentic matrix approach performed as well as the authentic analyte in surrogate matrix approach, indicating that the surrogate analyte approach is 
not required for the accurate quantification of endogenous compounds in complex samples [19].

To evaluation the four methods, four sets of calibration curve with QCs and 97 patient samples were analyzed using the four validated methods. The background subtraction approach has failed in the LOQs, calibration curve, and concentration calculation of patient samples. The first three methods have been successful in fulfilling all the criteria. The comparison between any two of the methods was carried out using the equation for sample reanalysis published in the EMEA/CHMP/EWP guideline on bioanalytical method validation, i.e. \%difference equals the absolute different value of two methods divided by the mean value of the two methods and multiplied by 100 . A RSD value of the three methods a calculated. The detailed comparison are shown in Figure 3. If the percent difference between the initial concentration and the concentration measured during the repeat analysis are not greater than $20 \%$ of their mean for at least $67 \%$ of the repeats, the EMEA guide considers the reproducibility of an analysis acceptable. The difference between any two methods or among the three methods presents $100 \%$ of samples with less than $20 \%$ for all the three amino acids. Mainly, the difference is under $10 \%$. According the EMEA criteria, the measured concentrations have no difference between or among the three methods. The BSA functioned effectively as a blank matrix for these three amino acids, considering cost, data quality, matrix similarity, and practicality.

\section{Conclusion}


The developed two-step sample preparation method using methanol as protein precipitation reagent in this study is simple and superior to other approaches. Four LCMS/MS methods have been developed for simultaneous determination of arginine, citrulline, and ornithine in human plasma. The four methods have been fully validated and compared. Three of them have been confirmed rapid and robust. The three methods have been directly applied to patient sample analysis. The results show that the measured concentrations have no difference between or among the three methods. When experimental data excluding its use as a blank matrix is absent, the BSA approach is the best choice among the four methods for an assay application, considering cost, data quality, matrix similarity, and practicality.

\section{References}

[1] J. Zagorski, M.R. Marchick, J.A. Kline, Rapid clearance of circulating haptoglobin from plasma during acute pulmonary embolism in rats results in HMOX1 upregulation in peripheral blood leukocytes, J. Thromb. Haemost., 8 (2010) 389-396.

[2] J.A. Watts, M.A. Gellar, M.B. Fulkerson, J.A. Kline, Pulmonary vascular reserve during experimental pulmonary embolism: effects of a soluble guanylate cyclase stimulator, BAY 41-8543, Crit. Care Med., 39 (2011) 2700-2704.

[3] J.A. Watts, M.A. Gellar, M.B. Fulkerson, S.K. Das, J.A. Kline, Arginase depletes plasma l-arginine and decreases pulmonary vascular reserve during experimental pulmonary embolism, Pulm. Pharmacol. Ther., 25 (2012) 48-54.

[4] J.A. Kline, J. Watts, D. Courtney, Y.Y. Lee, S. Hwang, Severe pulmonary embolism decreases plasma L-arginine, Eur. Respir. J., 43 (2014) 906-909.

[5] S.Z. Goldhaber, Venous thromboembolism: epidemiology and magnitude of the problem, Best Pract. Res. Clin. Haematol., 25 (2012) 235-242.

[6] J.A. Kline, M.R. Marchick, M.M. Hogg, Reduction in plasma haptoglobin in humans with acute pulmonary embolism causing tricuspid regurgitation, J. Thromb. Haemost., 7 (2009) 1597-1599.

[7] J.A. Kline, M.T. Steuerwald, M.R. Marchick, J. Hernandez-Nino, G.A. Rose, Prospective evaluation of right ventricular function and functional status 6 months after acute submassive pulmonary embolism: frequency of persistent or subsequent elevation in estimated pulmonary artery pressure, Chest, 136 (2009) 1202-1210. 
[8] J. Martens-Lobenhoffer, S.M. Bode-Boger, Mass spectrometric quantification of Larginine and its pathway related substances in biofluids: the road to maturity, $\mathrm{J}$. Chromatogr. B Analyt. Technol. Biomed. Life Sci., 964 (2014) 89-102.

[9] S. Shin, S.M. Fung, S. Mohan, H.L. Fung, Simultaneous bioanalysis of L-arginine, L-citrulline, and dimethylarginines by LC-MS/MS, J. Chromatogr. B Analyt. Technol. Biomed. Life Sci., 879 (2011) 467-474.

[10] J. Martens-Lobenhoffer, S. Postel, U. Troger, S.M. Bode-Boger, Determination of ornithine in human plasma by hydrophilic interaction chromatography-tandem mass spectrometry, J. Chromatogr. B Analyt. Technol. Biomed. Life Sci., 855 (2007) 271275.

[11] C.M. Brown, J.O. Becker, P.M. Wise, A.N. Hoofnagle, Simultaneous determination of $6 \mathrm{~L}$-arginine metabolites in human and mouse plasma by using hydrophilicinteraction chromatography and electrospray tandem mass spectrometry, Clin. Chem., 57 (2011) 701-709.

[12] J.W. Jones, G. Tudor, A. Bennett, A.M. Farese, M. Moroni, C. Booth, T.J. MacVittie, M.A. Kane, Development and validation of a LC-MS/MS assay for quantitation of plasma citrulline for application to animal models of the acute radiation syndrome across multiple species, Anal. Bioanal. Chem., 406 (2014) 4663-4675.

[13] N.C. van de Merbel, Quantitative determination of endogenous compounds in biological samples using chromatographic techniques, Trac-Trends in Analyt. Chem., 27 (2008) 924-933.

[14] W.L. Li, L.H. Cohen, Quantitation of endogenous analytes in biofluid without a true blank matrix, Anal. Chem., 75 (2003) 5854-5859.

[15] B.R. Jones, G.A. Schultz, J.A. Eckstein, B.L. Ackermann, Surrogate matrix and surrogate analyte approaches for definitive quantitation of endogenous biomolecules, Bioanalysis, 4 (2012) 2343-2356.

[16] P.K. Gupta, J. Brown, P.G. Biju, J. Thaden, N.E. Deutz, S. Kumar, M. HauerJensen, H.P. Hendrickson, Development of high-throughput HILIC-MS/MS methodology for plasma citrulline determination in multiple species, Anal. Methods, 3 (2011) 1759-1768.

[17] W. Lu, E. Kimball, J.D. Rabinowitz, A high-performance liquid chromatographytandem mass spectrometry method for quantitation of nitrogen-containing intracellular metabolites, J. Am. Soc. Mass Spectrom., 17 (2006) 37-50.

[18] A. Le, A. Ng, T. Kwan, K. Cusmano-Ozog, T.M. Cowan, A rapid, sensitive method for quantitative analysis of underivatized amino acids by liquid chromatographytandem mass spectrometry (LC-MS/MS), J. Chromatogr. B Analyt. Technol. Biomed. Life Sci., 944 (2014) 166-174.

[19] S. Ongay, G. Hendriks, J. Hermans, M. van den Berge, N.H. ten Hacken, N.C. van de Merbel, R. Bischoff, Quantification of free and total desmosine and isodesmosine in human urine by liquid chromatography tandem mass spectrometry: a comparison of the surrogate-analyte and the surrogate-matrix approach for quantitation, J. Chromatogr. A, 1326 (2014) 13-19. 


\section{Figure Legends}

Figure 1. Comparing the effect of reagent type and volume on the recoveries of all the three amino acids. Multiple volumes $(2,3,4,5,7,9$, and 11 times of $50 \mu \mathrm{L})$ of acetonitrile (ACN), isopropanol (IPA), and methanol $(\mathrm{MeOH})$ were used to precipitate proteins in $50 \mu \mathrm{L}$ of plasma spiked with arginine (AR21), citrulline (Cl41), ornithine (OR61), and their internal standards (AR61, Cl21, and OR21). It shows that reagent type is a greater factor than volume for the recovery and volume is a critical factor as well. The performance of methanol is the best for all the three amino acids.

Figure 2. Representative chromatograms of the current LC-MS/MS analysis using the chosen transitions from a patient sample spike with mid QC and IS. Peak intensity and elution times (min) are shown for authentic analytes (AR01, CI01, and OR01), surrogate analytes (AR21, Cl41, and OR61), and internal standards (AR61, Cl21, and OR21).

Figure 3. Comparing the performance of the three four methods: water as blank matrix, 1\% BSA in PBS as blank matrix, and surrogate analyte. Percentage difference between any two of the methods and RSD value among the three methods were carried out. The results show that he difference between any two methods or among the three methods presents $100 \%$ of samples with less than $20 \%$ for all the three amino acids. Mainly, the difference is under $10 \%$. Therefore, the measured concentrations in 97 human plasma samples are considered not different between or among the three methods. 


\section{Table}

Table 1. Precisions and accuracies of calibration standards of OR01 in 1\% BSA from three validation batches.

\begin{tabular}{|c|c|c|c|c|c|c|c|c|}
\hline \multirow[t]{3}{*}{ Analysis group } & \multicolumn{8}{|c|}{ Theoretical concentration $(\mu \mathrm{M})$} \\
\hline & 4.0 & 5.0 & 10.0 & 25.0 & 75.0 & 100.0 & 150.0 & 200.0 \\
\hline & \multicolumn{8}{|c|}{ Measured concentration $(\mu \mathrm{M})$} \\
\hline 001 & 4.1 & 4.9 & 9.8 & 25.5 & 76.0 & 96.3 & 159.5 & 192.9 \\
\hline 002 & 3.9 & 4.8 & 10.1 & 25.1 & 79.5 & 101.1 & 150.6 & 193.9 \\
\hline 003 & 4.2 & 4.9 & 10.0 & 24.2 & 76.2 & 98.5 & 153.7 & 197.3 \\
\hline $\mathrm{n}$ & 3 & 3 & 3 & 3 & 3 & 3 & 3 & 3 \\
\hline Mean & 4.1 & 4.9 & 10.0 & 24.9 & 77.2 & 98.6 & 154.6 & 194.7 \\
\hline RSD (\%) & 3.8 & 1.2 & 1.5 & 2.7 & 2.5 & 2.4 & 2.9 & 1.2 \\
\hline Accuracy (\%) & 101.7 & 97.3 & 99.7 & 99.7 & 103.0 & 98.6 & 103.1 & 97.4 \\
\hline
\end{tabular}


Table 2. Precisions and accuracies of quality control samples for OR01 in 1\% BSA from three validation batches.

\begin{tabular}{|c|c|c|c|c|c|}
\hline \multirow{2}{*}{$\begin{array}{l}\text { Analysis } \\
\text { group }\end{array}$} & \multirow[t]{2}{*}{ Statistics } & \multicolumn{4}{|c|}{ Theoretical concentration $(\mu \mathrm{M})$} \\
\hline & & 4.0 & 7.5 & 50.0 & 125.0 \\
\hline \multirow{4}{*}{001} & $\mathrm{n}$ & 6 & 6 & 6 & 6 \\
\hline & Intra-assay mean & 4.1 & 7.6 & 47.7 & 130.1 \\
\hline & RSD (\%) & 1.6 & 1.9 & 1.1 & 1.6 \\
\hline & Accuracy (\%) & 102.5 & 101.1 & 95.4 & 104.1 \\
\hline \multirow{4}{*}{002} & $\mathrm{n}$ & 6 & 6 & 6 & 6 \\
\hline & Intra-assay mean & 4.1 & 7.3 & 47.4 & 127 \\
\hline & RSD (\%) & 1.6 & 2.2 & 1.2 & 2.6 \\
\hline & Accuracy (\%) & 102.4 & 97.2 & 94.7 & 101.6 \\
\hline \multirow{4}{*}{003} & $\mathrm{n}$ & 6 & 6 & 6 & 6 \\
\hline & Intra-assay mean & 4.1 & 7.6 & 47.6 & 126 \\
\hline & RSD (\%) & 2.0 & 2.7 & 1.8 & 1.3 \\
\hline & Accuracy (\%) & 103.5 & 102.0 & 95.2 & 100.8 \\
\hline \multirow{4}{*}{ Overall } & $\mathrm{n}$ & 18 & 18 & 18 & 18 \\
\hline & Inter-assay mean & 4.1 & 7.5 & 47.5 & 127.7 \\
\hline & RSD (\%) & 1.8 & 3.0 & 1.4 & 2.3 \\
\hline & Accuracy (\%) & 102.8 & 100.0 & 95.1 & 102.2 \\
\hline
\end{tabular}


Table 3. Recoveries at low, mid and high QC levels for AR01 (IS, AR61), CI01 (IS, $\mathrm{Cl} 21)$, and OR01 (IS, OR21) in 1\% BSA.

\begin{tabular}{llcccccc}
\hline QC levels & Statistics & AR01 & AR61 & CI01 & CI21 & OR01 & OR21 \\
\hline \multirow{2}{*}{ Low } & & & & & & & \\
& RSD (\%) & $4.7 / 2.2$ & $4.2 / 1.2$ & $7.3 / 12.9$ & $6.3 / 10.2$ & $3.9 / 3.4$ & $3.0 / 0.3$ \\
& Recovery (\%) & 83.0 & 87.1 & 90.6 & 91.1 & 85.1 & 87.9 \\
\multirow{2}{*}{ Mid } & RSD (\%) & $6.1 / 0.7$ & $6.6 / 0.6$ & $8.7 / 5.7$ & $8.8 / 5.9$ & $2.9 / 1.7$ & $3.7 / 1.3$ \\
& Recovery (\%) & 84.5 & 94.1 & 86.0 & 96.6 & 80.8 & 91.5 \\
& & & & & & & \\
\multirow{2}{*}{ High } & RSD (\%) & $4.3 / 6.1$ & $4.5 / 6.7$ & $11.8 / 12.9$ & $12.1 / 11.5$ & $2.8 / 5.0$ & $3.6 / 4.5$ \\
& Recovery (\%) & 87.3 & 93.4 & 87.3 & 93.7 & 84.1 & 92.0 \\
& & & & & & & \\
Overall & Recovery (\%) & 85.0 & 91.5 & 88.0 & 93.8 & 83.3 & 90.4 \\
\hline
\end{tabular}

a RSD: RSD of six replicates of extracted QC samples/RSD of three replicates of recovery samples. 
Table 4. Stability of AR01, CIO1, and OR01 at low, mid and high QC levels in 1\% BSA under different conditions.

\begin{tabular}{|c|c|c|c|c|}
\hline QC levels & Statistics & AR01 & $\mathrm{Cl} 01$ & OR01 \\
\hline \multicolumn{5}{|c|}{ Three freeze/thaw matrix stability } \\
\hline & $\mathrm{n}$ & 6 & 6 & 6 \\
\hline \multirow[t]{3}{*}{ Low } & RSD (\%) & 2.0 & 2.1 & 2.3 \\
\hline & Accuracy (\%) & 99.1 & 102.0 & 103.6 \\
\hline & $\mathrm{n}$ & 6 & 6 & 6 \\
\hline \multirow[t]{3}{*}{ Mid } & RSD (\%) & 1.6 & 1.9 & 1.5 \\
\hline & Accuracy (\%) & 95.1 & 97.6 & 97.2 \\
\hline & $\mathrm{n}$ & 6 & 6 & 6 \\
\hline \multirow[t]{2}{*}{ High } & RSD (\%) & 2.5 & 1.3 & 1.6 \\
\hline & Accuracy (\%) & 98.2 & 103.2 & 102.4 \\
\hline \multicolumn{5}{|c|}{$48 \mathrm{~h}$ room temperature matrix stability } \\
\hline & $\mathrm{n}$ & 6 & 6 & 6 \\
\hline \multirow[t]{3}{*}{ Low } & RSD (\%) & 0.7 & 2.8 & 1.4 \\
\hline & Accuracy (\%) & 97.8 & 102.2 & 100.9 \\
\hline & $\mathrm{n}$ & 6 & 6 & 6 \\
\hline \multirow[t]{3}{*}{ Mid } & RSD (\%) & 1.7 & 1.5 & 1.1 \\
\hline & Accuracy (\%) & 95.5 & 97.9 & 95.3 \\
\hline & $\mathrm{n}$ & 6 & 6 & 6 \\
\hline \multirow[t]{2}{*}{ High } & RSD (\%) & 1.3 & 0.6 & 1.6 \\
\hline & Accuracy (\%) & 96.7 & 100.9 & 100.0 \\
\hline \multicolumn{5}{|c|}{55 days -60 to $-80 \circ \mathrm{C}$ matrix stability } \\
\hline & $\mathrm{n}$ & 6 & 6 & 6 \\
\hline \multirow[t]{3}{*}{ Low } & RSD (\%) & 1.9 & 11.3 & 3.9 \\
\hline & Accuracy (\%) & 95.3 & 98.4 & 99.3 \\
\hline & $\mathrm{n}$ & 6 & 6 & 6 \\
\hline \multirow[t]{3}{*}{ Mid } & RSD (\%) & 2.0 & 3.0 & 1.2 \\
\hline & Accuracy (\%) & 97.5 & 99.9 & 100.4 \\
\hline & $\mathrm{n}$ & 6 & 6 & 6 \\
\hline
\end{tabular}


3.4

2.8

2.8

Accuracy (\%)

98.2

102.8

102.9 